\title{
The Effects of Performing a One-legged Bridge with Hip Abduction and Unstable Surface on Trunk and Gluteal Muscle Activation in Healthy Adults
}

\author{
Jong-Woo Bak ${ }^{1}$, Min-Kwon Cho ${ }^{1}$, Yi-Jung Chung ${ }^{2}$ \\ 'Department of Physical Therapy, The graduate school, Sahmyook University, Seoul; ${ }^{2}$ Department of Physical Therapy, College of Health and Welfare, \\ Sahmyook University, Seoul, Korea
}

Purpose: This study investigated the influence of muscle activity of the trunk and lower limb during a bridge exercise using a unstable surface and during one-legged bridge hip abduction in healthy adults.

Methods: Nineteen healthy participated in this study (12 males and 7 females, aged 29.0 \pm 5.0). The participants were instructed to perform the bridge exercises under six different conditions. Trunk and lower limb muscle activation, such as the erector spinae (ES), gluteus maximus (GM), external oblique (EO), and internal oblique (IO), was measured using surface electromyography. The six different bridge exercise conditions were conducted randomly. Data analysis was performed by using the mean scores after three trials of each condition. Results: On the ipsilateral side, muscle activity of the IO, EO, and ES during the hip abduction condition (Single-legged hip abduction bridge, Bridge with use of a ball and single-leg hip abduction, Bridge with use of a sling and single-leg hip abduction) was significantly higher than those during Unstable surface (Bridge with use of a ball, Bridge with use of a sling) and General bridging exercise ( $p<0.05$ ). In the contralateral side, activities of the GM and EO during Single-legged hip abduction bridge, Bridge with use of a ball and single-leg hip abduction and Bridge with use of a sling and single-leg hip abduction was significantly higher than that during Bridge with use of a ball, Bridge with use of a sling and General bridging exercise $(p<0.05)$.

Conclusion: This study demonstrated that performing a bridge exercise with use of a sling and single-leg hip abduction had an effect on trunk and gluteal muscle activation. The findings of this study suggest that this training method can be clinically effective for unilateral training and for patients with hemiplegia.

Keywords: Bridge exercise, Surface electromyography, Hip abduction, Unstable surface

\section{INTRODUCTION}

The abdominal muscles contribute to providing high mechanical stability to the trunk, which many clinicians have been brought to attention. ${ }^{1}$ In order to maintain trunk stability, co-contraction of the abdominal musculature is needed, and the nervous system plays an important role in producing trunk stability by regulating the appropriate intensity of control of the nervous system to produce a coordinated action of the trunk muscles in various situations. ${ }^{2}$ The trunk muscles that control the trunk produces an upright posture of the spine, maintains balance, and also provides adequate amount

Received May 30, 2016 Revised Jun 22, 2016

Accepted Jun 27, 2016

Corresponding author Yi-Jung Chung

E-mail yijung36@syu.ac.kr of coordination of the head and trunk so that movement may occur in harmony earlier on, and at the same time provide flexibility and rigidity (stiffness) while walking to produce a smooth gait pattern. ${ }^{3}$

Bridge exercises are considered one of the basic exercises that can be performed on a mat that are not only useful for improving trunk stabilization, providing pressure relief to the gluteal area, promoting ease of performance of bed mobility and bed pan use, permitting lower body dressing, developing the ability to make postural adjustments during sit-to-stand, facilitating pelvic movement, but it is also related to pelvic and functional movements involved during walking.
Copylight (C2016 The Korea Society of Physical Therapy

This is an Open Access article distribute under the terms of the Creative Commons Attribution Non-commercial License (Http:// creativecommons.org/license/by-nc/4.o.) which permits unrestricted non-commercial use, distribution, and reproduction in any medium, provided the original work is properly cited. 
The purpose of bridge exercises are to prevent damage occurring to the spinal muscles, joints, ligaments and tissues and are commonly used to promote stability. ${ }^{5}$ In addition, bridge exercises allows weight-bearing to occur through bilateral lower extremities, develops the ability to make postural adjustments during sit-tostand, and strengthens the lower spine and hip extensor muscles during the stance phase of gait. ${ }^{6}$

Bridge exercises have been studied in various forms. Many studies have included the use of not only the general bridge exercise, but also those including bridge exercises with lower extremity elevation, unstable surfaces, slings, and balls. ${ }^{7}$ It is being reported in the literature an increase in trunk muscle activity when performing stabilization exercises with the use of a sling or cushion to provide an unstable surface. ${ }^{8-10}$ And similar results of increased muscle activity are being shown with bridge exercises being performed on an unstable surface compared to the general bridge performance. ${ }^{11}$ A significant increases in multifidus (MF) and erector spinae muscle activity has been reported when performing a bridge exercise with the lower extremity placed in a sling compared to placing it on a ball or on the floor. ${ }^{12}$ Training with the use of lower extremity elevation is being reported in many studies. ${ }^{11,13-15}$ Most studies state that ipsilateral trunk muscle activity is increased when performing a bridge exercise including lower extremity elevation compared to performing a general bridge. ${ }^{13,14}$ Park et al. ${ }^{16}$ has reported that an increase in internal oblique (IO) and MF muscle activity was exhibited when subjects performed bridge exercises with lower extremity movements (adduction, abduction) compared to the general bridge exercise. Jang et al. ${ }^{17}$ has reported that increases in trunk muscle activity can been observed with applying hip adduction to a bridge exercise compared to performing a general bridge exercise. Although studies on the effects of lower extremity movements being included with bridge exercises are being actively conducted, studies focusing on the use of hip abduction are lacking. It has been confirmed that most previous studies have investigated the effects of the use of lower extremity elevation or unstable surfaces during bridge exercises on muscle activation.

Jang et al. ${ }^{17}$ conducted a study comparing the difference between a general bridge and a bridge with hip adduction, however, the study did not include the use of an unstable surface. Park et al ${ }^{16}$ conducted a study on bridge exercises with the use of a sling, but did not compare with the general bridge or other degrees of hip adduction.
In addition, there are a lack of studies that investigates bridge exercises with the use of an unstable surface and hip abduction and the use of surface electromyography.

Therefore, the purpose of this study was to investigate the effects of bridge exercise performance on an unstable surface and with the inclusion of hip abduction on trunk and gluteal muscle activity, as well as to discover which type of bridge exercise would most efficiently activate the gluteal muscles in healthy adults.

\section{METHODS}

\section{Subjects}

This was a cross-sectional study. Nineteen subjects have voluntarily agreed to participate in the study after being informed of the purpose, methods, use of results of the study. In this study, healthy adults were connected to a surface electromyography (EMG) to measure the appropriate muscle activation patterns during the performance of a general bridge, single-legged hip abduction bridge, bridge with use of a ball, bridge with use of a ball and single-leg hip abduction, bridge with use of a sling, bridge with use of a sling and single-leg hip abduction in random order.

Healthy subjects in their twenties and thirties had voluntarily agreed to participate in the study after being fully informed about the study. Subjects were excluded if they had experiences of back pain for the last 6 months, congenital limb deformities, orthopedic or serious neurological diseases, trauma, or pain (Table 1).

\section{Experimental methods}

\section{1) Research procedure}

The maximum voluntary isometric contraction (MVIC) of the subjects was assessed from the erector spinae (ES), external oblique (EO), internal oblique (IO), and gluteus maximus (GM). Prior to the intervention, subjects were fully informed of the various bridge conditions as well given the opportunity to practice each condition

Table 1. General characteristics of subjects

\begin{tabular}{lc}
\hline & Subjects \\
\hline Sex (male/female) & $19(12 / 7)$ \\
Age (year) & $29.0 \pm 5.0^{\mathrm{a}}$ \\
Height $(\mathrm{cm})$ & $171.4 \pm 7.1$ \\
Weight $(\mathrm{kg})$ & $66.0 \pm 15.1$ \\
\hline
\end{tabular}

aMean $\pm S D$. 
three times.

The following bridge conditions are described below. Each condition was performed randomly with three trials per condition.

\section{(1) General bridge}

Patients were positioned in a supine hook-lying position with 90 de- grees of knee flexion. Patients were instructed to lift their hips while maintaining it in 0 degrees (Figure 1).,17

\section{(2) Single-legged hip abduction bridge}

Starting with the general bridge position, the left lower extremity maintained 90 degrees of knee flexion while the right lower extrem-
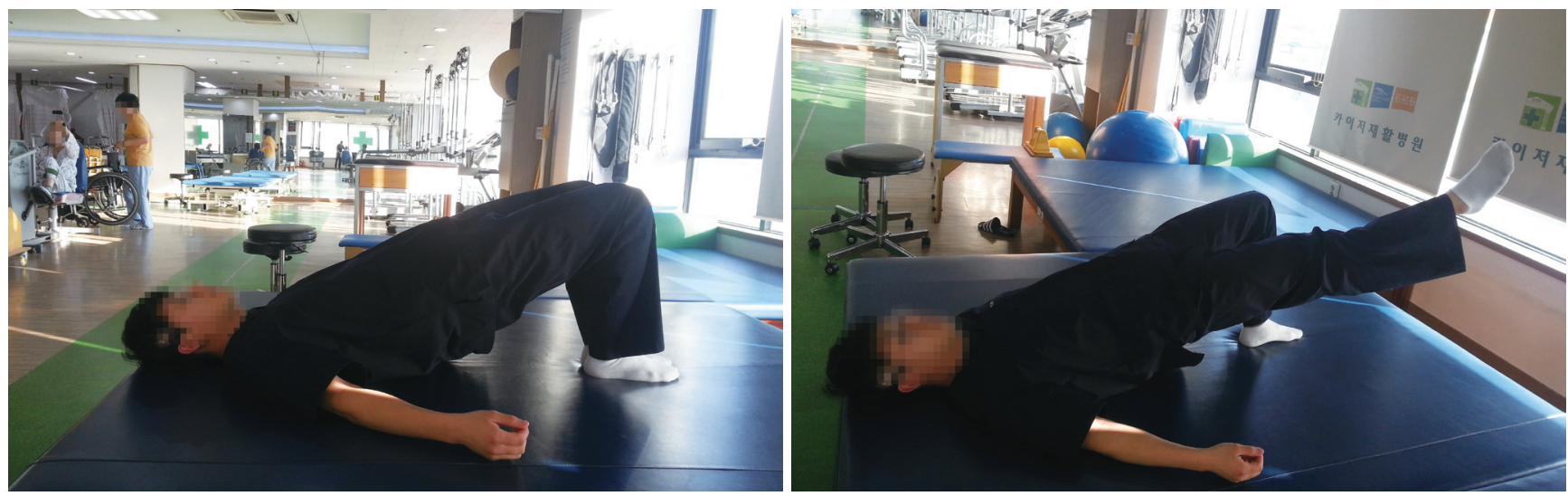

Figure 1. General Bridge, bridge with single-leg hip abduction.
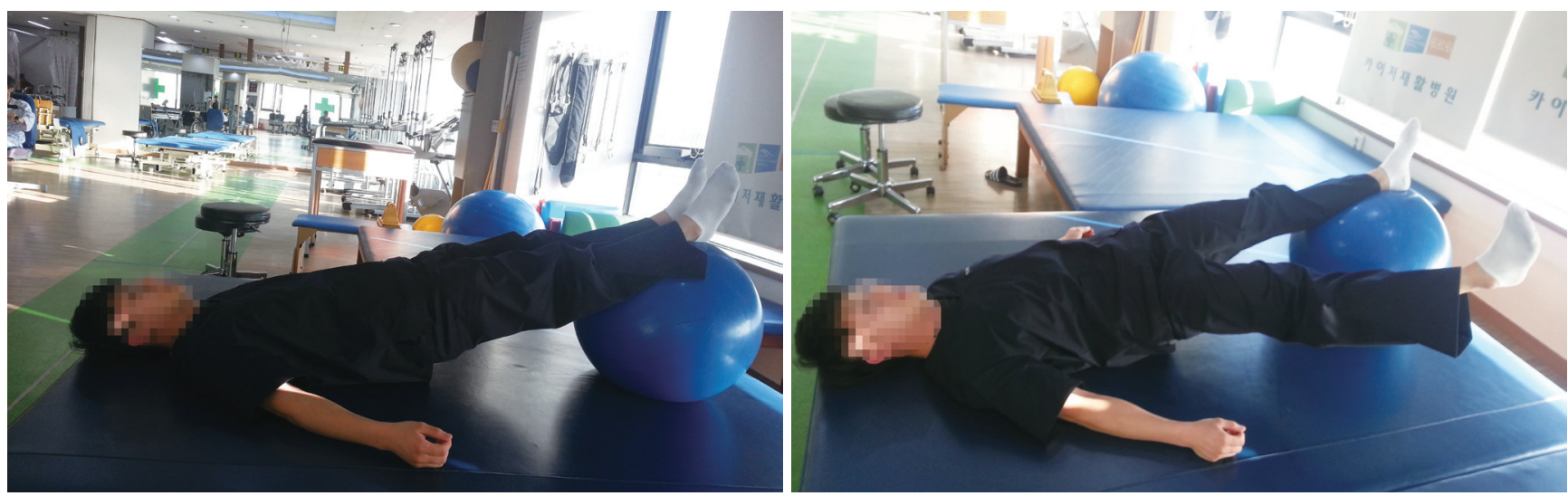

Figure 2. Bridge with ball, bridge with use of a ball and single-leg hip abduction
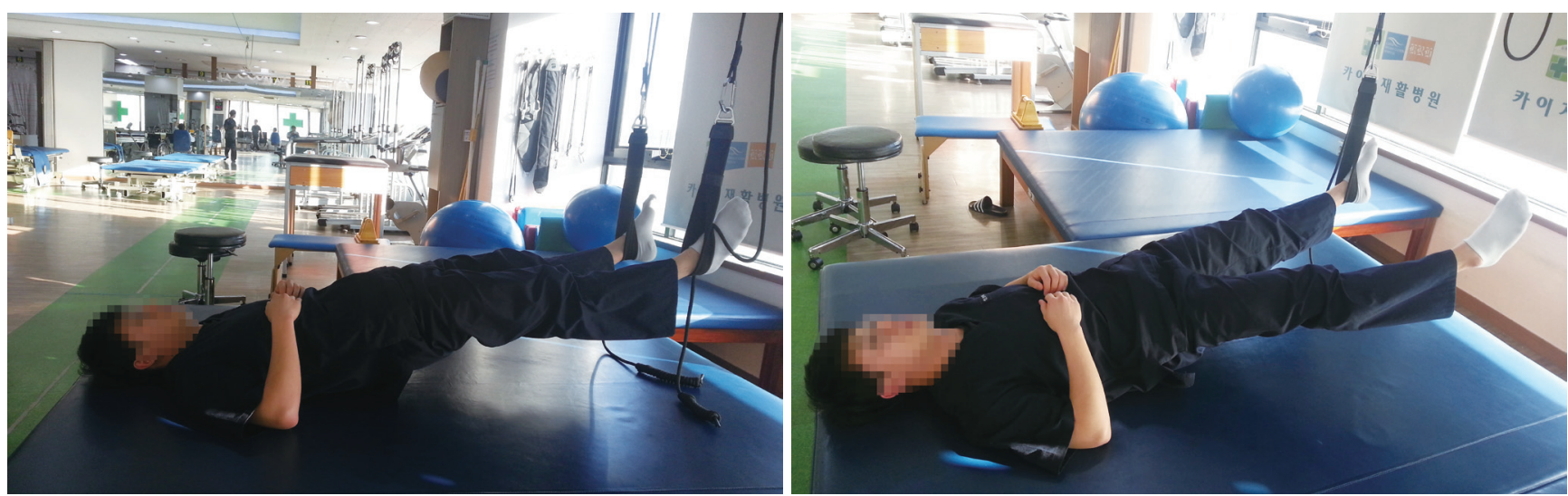

Figure 3. Bridge with sling, bridge with use of a sling and single-leg hip abduction. 
ity was elevated with $30^{\circ}$ of hip abduction. In this position, the subject was instructed to raise the buttocks from the floor and maintain the hip joint in $0^{\circ}$.

Beginning with the same starting position as the general bridge, the left knee is maintained at 90 degrees of flexion, while the right lower extremity is lifted and abducted to 30 degrees, followed by raising of the hip while maintaining 0 degrees of hip flexion (Figure 1).

\section{(3) Bridge exercise with use of a ball}

Starting from a supine position with bilateral ankles placed onto a ball that is $65 \mathrm{~cm}$ in diameter, subjects were instructed to lift the buttocks from the floor until they reached $0^{\circ}$ of the hip joint (Figure 2).

\section{(4) Bridge with use of a ball and single-leg hip abduction}

Starting from a supine position with bilateral ankles placed onto a ball that is $65 \mathrm{~cm}$ in diameter, and the right lower extremity elevated and hip abducted to $30^{\circ}$, subjects were instructed to lift the buttocks from the floor until they reached $0^{\circ}$ of the hip joint (Figure 2).

\section{(5) Bridge with use of a sling}

Starting from a supine position and bilateral ankles placed in a sling at knee height, subjects were instructed to lift the buttocks until they reached $0^{\circ}$ of the hip joint (Figure 3 ).

\section{(6) Bridge with use of a sling and single-leg hip abduction}

Starting from a general bridge position, the left lower extremity has been placed onto a sling at knee-height at the ankle and the right lower extremity was elevated and placed into $30^{\circ}$ of hip abduction. In this position, subjects were instructed to lift the buttocks from the floor until they reached $0^{\circ}$ of the hip joint (Figure 3 ).

The subjects performed the bridge exercises following the signals of a therapist. Subjects were instructed to raise the buttocks from the floor until they've reached $0^{\circ}$ of the hip joint and were asked to maintain the position for 5 seconds. ${ }^{2}$ Subjects performed each bridge condition three times, and were provided with a 1 minute rest period between each condition. The bridge exercises and measurements were performed in random order.

\section{2) Measurement}

\section{(1) Collection of EMG materials}

To investigate the muscle activation patterns during the bridge con- ditions, a Telemyo 2400 G2 Telemetry EMG system (Noraxon, USA, 2011) was used. A sampling rate of $100 \mathrm{~Hz}$ and a bandpass filter 10-450 Hz was used. The electrode placement sites for the erector spinae was the iliac crest and $2 \mathrm{~cm}$ inside the triangle that connects the lower ribs, $15 \mathrm{~cm}$ above the umbilicus for the external oblique, between the sacrum and greater trochanter for the gluteus maximus, and $2 \mathrm{~cm}$ medial to the anterior superior iliac spine for the internal oblique. ${ }^{19}$

Prior to the experiment, the maximum voluntary isometric contraction (MVIC) data collected from the ES, EO, IO, and GM was conducted for 5 seconds each. The MVIC of the ES was assessed with the subject lying in prone position and raising the trunk off the treatment table while a therapist stabilized the lower extremities. The MVIC of the IO and EO was assessed with the subject in supine position and lifting the trunk in a diagonal direction while a therapist stabilized the lower extremities. The MVIC of the GM was assessed with the subject in prone position and raising both lower extremities while a therapist stabilized the upper extremities. ${ }^{18}$ The electrode placement sites were cleaned with alcohol and shaved to minimize the resistance on the skin. ${ }^{15}$

\section{(2) Data and statistical analysis}

EMG measurement of muscle activity was performed using the Myoresearch XP Master edition software (Noraxon Inc, Arizona, USA, 2011). After the data was analysed with full wave rectification and calculated to root mean square, and the MVIC value was normalized to a percentage value. Once the buttocks were raised from the table per bridge condition, measurements were taken for 5 seconds as soon as the hip joint and trunk were in alignment. ${ }^{11,19}$ Data from the first and last second were excluded, while data from the middle three seconds were analyzed. After all of the conditions were repeated and measured three times and the mean values were obtained.

Data was collected using the PASW version 19.0 (SPSS Inc., Chicago, USA).

Descriptive statistics was used to analyze the general characteristics of subjects, and a one-way repeated measure ANOVA was used to investigate for the effects of various bridge exercise conditions on trunk and lower extremity muscle activity. To examine for the differences in trunk and lower extremity muscle activity, data was analyzed using the least significant difference (LSD). The significance 
level was set at $\mathrm{p}<0.05$.

\section{RESULTS}

\section{A comparison of ipsilateral trunk and lower extremity muscle activation according to various bridge condition}

For the ipsilateral ES, muscle activity was significantly greater during bridge exercise with hip abduction, bridge with use of a ball, bridge with use of a ball and single-leg hip abduction, bridge with use of a sling, and bridge with use of a sling with single-leg hip abduction compared with the general bridge condition $(\mathrm{p}<0.05)$. Muscle activity was significantly greater during bridge with use of a ball and single-leg hip abduction and sling with single-leg hip abduction compared with the single-leg hip abduction bridge conditions $(\mathrm{p}<0.05)$.

For the Ipsilateral EO muscle activity, muscle activity was the most significantly increased during the bridge with use of a sling and single-leg hip abduction compared with all of the other bridge conditions, except for bridge with use of a ball and single-leg hip abduction condition.

For the ipsilateral IO, although muscle activity was low with the bridge with use of a sling condition, there was no significant difference compared with the general bridge and bridge with use of a ball conditions (Table 2).

For both ipsilateral EO and IO, muscle activity was significantly greater during the performance of a bridge with use of a sling and single-leg hip abduction compared to all of the other bridge conditions, except for the bridge with use of a ball and single-leg hip abduction $(\mathrm{p}<0.05)$.

\section{A comparison of the contralateral trunk and lower extremity} muscle activation according to various bridge conditions

For the contralateral ES, muscle activity was the greatest during the bridge with use of a ball and was significantly greater compared with the general bridge, single-leg hip abduction, bridge with use of a ball and single-leg hip abduction, bridge with use of a sling and single-leg hip abduction conditions $(\mathrm{p}<0.05)$.

For the contralateral GM, the greatest amount of muscle activity was observed with the single-leg hip abduction condition, and was significantly greater compared with the general bridge, bridge with use of a ball, bridge with use of a ball and single-leg hip abduction, and bridge with use of a sling condition $(\mathrm{p}<0.05)$.

For the contralateral EO, the greatest amount of muscle activity was observed during the bridge with use of a sling and single-leg hip abduction condition, and was significantly greater compared with the general bridge, single-leg hip abduction, bridge with use of a ball, bridge with use of a sling conditions $(\mathrm{p}<0.05)$. There was no significant difference between the bridge with single-leg hip abduction and bridge with use of a ball and single-leg hip abduction conditions.

For the contralateral IO, muscle activity was the greatest during the single-leg hip abduction condition, and was significantly greater compared with the general bridge, bridge with use of ball, and bridge with use of a sling conditions $(\mathrm{p}<0.05)$. There was no significant difference between the bridge with single-leg abduction, bridge with use of a ball and single-leg hip abduction, and bridge with use of a sling and single-leg hip abduction conditions (Table 2).

Table 2. Comparison of the trunk and lower extremity activity during bridge exercise (Unit: \%MVIC)

\begin{tabular}{|c|c|c|c|c|c|c|c|c|}
\hline & \multicolumn{2}{|c|}{ Erector Spinae } & \multicolumn{2}{|c|}{ Gluteus Maximus } & \multicolumn{2}{|c|}{ External Oblique } & \multicolumn{2}{|c|}{ Internal Oblique } \\
\hline & Ipsilateral & Contralateral & Ipsilateral & Contralateral & Ipsilateral & Contralateral & Ipsilateral & Contralateral \\
\hline Whe & $41.60 \%(14.18)$ & 30) & $.42 \%(6.52)$ & 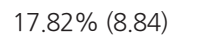 & $43 \%(5.96)$ & $0.40(1.75)$ & (I. I. & $.00)$ \\
\hline $\mathrm{HA}$ & $48.92 \%(15.27)^{\star}$ & $7 \%(17.43)$ & $6(3.24)^{*}$ & $9 \%(19.42)^{*}$ & $\%(14.76)^{*}$ & $\%(13.19)^{*}$ & $66 \%(28.17)^{*}$ & $16)^{*}$ \\
\hline BB & $49.17 \%(15.48)^{\star}$ & $49.38 \%(16.97)^{\star+}$ & $6.56 \%(3.24)^{\star}$ & $7.86 \%(5.17)^{\star}$ & $8.26 \%(6.42)^{+}$ & $8.88 \%(8.46)^{+}$ & $10.50 \%(10.66)^{+}$ & $10.80 \%(7.73)^{+}$ \\
\hline BBHA & $56.50 \%(18.97)^{\star+}$ & $40.13 \%(16.77)^{+}$ & $6.17 \%(3.53)^{\star}$ & $24.81 \%(12.44)^{\star+\neq}$ & $19.11 \%(12.06)^{k+}$ & $15.62 \%(12.58)^{\star *}$ & $33.08 \%(30.62)^{\star *}$ & $14.07 \%(8.76)^{*+}$ \\
\hline BS & $47.09 \%(12.47)^{\star s}$ & $47.08 \%(15.48)^{+\S}$ & $15.67 \%(10.69)^{++\$}$ & $15.75 \%(11.06)^{++\xi}$ & $6.09 \%(4.31)^{x+15}$ & $7.19 \%(5.93)^{+\S}$ & $8.86 \%(9.98)^{+\S}$ & $10.96 \%(12.06)$ \\
\hline BSHA & $62.27 \%(18.28)^{x+58 / /}$ & $42.81 \%(19.90)^{\dagger / /}$ & $6.66 \%(3.52)^{\star / /}$ & $35.36 \%(11.68)^{\star \star \$ / / /}$ & $22.30 \%(17.40)^{x+1 / /}$ & $16.54 \%(11.61)^{* \neq / 1 /}$ & $37.57 \%(34.07)^{\star+\neq / /}$ & $12.96 \%(9.03)^{\star}$ \\
\hline$F(p)$ & $12.42(<0.001)$ & $4.72(0.01)$ & $22.40(<0.001)$ & $32.40(<0.001)$ & $24.69(<0.001)$ & $14.77(<0.001)$ & $19.38(<0.001)$ & $3.50(0.006)$ \\
\hline
\end{tabular}

Values are presented as $n$ (\%) or mean (SD).

GBR: General bridging exercise, SHA: Single-legged hip abduction bridge, BB: Bridge with use of a ball, BBHA: Bridge with use of a ball and single-leg hip abduction, BS: Bridge with use of a sling, BSHA: Bridge with use of a sling and single-leg hip abduction.

*Statistically significant difference with GBR ( $p<0.05)$; +Statistically significant difference with SHA ( $p<0.05)$; *Statistically significant difference with BB ( $p<0.05)$; $\$$ Statistically significant difference with BBHA ( $p<0.05)$; "Statistically significant difference with BS $(p<0.05)$. 


\section{DISCUSSION}

This study investigated the effects of performing general bridge exercises and bridge exercises using an unstable surface as well as hip abduction on trunk and lower extremity muscle activation by comparing the muscle activity of the ES, EO, IO, and GM using surface EMG in healthy adults.

It has been confirmed that when performing bridge exercises with single-leg hip abduction, it created a significantly greater amount of ipsilateral ES, bilateral EO and IO, and contralateral GM muscle activity and a significantly lesser amount in the ipsilateral GM compared with performing bride exercises on an unstable surface. It can be stated that with a single-leg hip abduction bridge, the base of support is decreased and creates instability, and therefore, the muscle activity of the trunk becomes increased for compensations. ${ }^{20}$ Also, muscle activity of the contralateral trunk and lower extremity increases when the ipsilateral lower extremity has been raised in order to maintain the position and with stand the weight being lifted by the ipsilateral side. ${ }^{21}$

The instability that is created when performing a bridge exercise with one lower extremity elevated leads to co-contraction of muscles in order to overcome the instability and maintain balance. ${ }^{20}$

This study has confirmed that there was a significant increase in muscle activity of the ipsilateral ES, EO, and IO during the performance of a bridge with use of a sling and single-leg hip abduction compared with a single-leg hip abduction bridge and bridge with use of a sling conditions. Bolgla and $\mathrm{Uh}^{22}$ compared three closedchain and three open-chain exercises and reported that there was a greater increase in muscle activation during a closed-chain hip abduction exercise in a side-lying position $(\mathrm{p}<0.05)$. The length of the lever arm plays an important role in optimizing muscle activation. An increase in lever arm leads to an increase in the mechanical use and strength of muscles. It has been observed in this study that there was an increase in ipsilateral muscle activation during the single-legged bridge with use of a sling compared with the general and single-legged bridge conditions, and placing the contralateral ankle in a sling while using the ipsilateral lower extremity to raise the body produced an increase in lever arm compared with the general bridge position. The increase in muscle activation was considered to be due to the increase in lever arm. Haynes ${ }^{23}$ conducted a study where muscle activation was assessed after subjects had performed exercises on various unstable conditions and had found that greater instability levels produced greater muscle activation of the entire body. The increase in muscle activity of the ipsilateral ES, EO, and $\mathrm{IO}$ is predicted to have occurred due to a decrease in stability caused by a single-leg sling bridge exercise compared with a single-leg bridge exercise.

It has been observed from this study that there was a significant increase in muscle activity of the ES, EO, and $\mathrm{IO}$, as well as a greater significant increase in contralateral GM compared with the ipsilateral GM during the performance of a bridge exercise with single-leg hip abduction compared with the general bridge and bridge exercises performed on an unstable surface. These findings are in agreement from previous studies. Park et al. ${ }^{16}$ conducted a study on healthy adults and observed a greater increase in trunk muscle activity during the performance of a bridge exercise with use of a sling in addition to hip abduction and adduction compared with a bridge exercise with use of a sling. Susan et al. ${ }^{7}$ reported a significant increase in muscle activity during the performance of a bridge with use of a sling and hip abduction compared with a bridge with hip abduction exercise.

The use of a small number of healthy subjects would make it difficult to make any generalizations. Due to having to perform 6 different bride exercises, it is possible that a learning effect could have occurred. In addition, further studies that are not cross-sectional, and that will examine changes in muscle thickness, strength, and fatigue factors through strength training and its effects on trunk and lower extremity muscles are necessary.

This study demonstrated that performing a bridge exercise with use of a sling and single-leg hip abduction had an effect on trunk and gluteal muscle activation. The findings of this study suggest that this training method can be clinically effective for unilateral training and for patients with hemiplegia.

\section{REFERENCES}

1. Kim MH, Kim YS, Yoon BC. Gender difference in trunk stability and standing balance during unexpected support surface translation in healthy adults. J Kor Phys Ther. 2014:26(2):97-103

2. Jung KS, Chung YJ. Effects of the support surface condition on muscle activity of trunk muscles during weight shifting exercise. J Kor Phys Ther. 2012:24(5):300-5

3. Jang SH, Ann JH, Kim JS. Effect of trunk control training on labile surface on relative impulse in the persons with stroke. J Kor Phys Ther. 
2012:24(2):163-9

4. O’Sullivan SB, Schmitz TJ, Fulk G. Physical rehabilitation. Philadelphia: FA Davis. 2014:709.

5. Kisner C, Colby LA. Therapeutic exercise: Foundations and techniques. Philadelphia, FA Davis, 2002:383-480

6. O'Sullivan PB. Lumbar segmental 'instability': Clinical presentation and specific stabilizing exercise management. Man Ther. 2000;5(1):2-12.

7. Saliba SA, Croy T, Guthrie R et al. Differences in transverse abdominis activation with stable and unstable bridging exercises in individuals with low back pain. N Am J Sports Phys Ther. 2010;5(2):63-73.

8. Escamilla RF, Lewis C, Bell D, et al. Core muscle activation during Swiss ball and traditional abdominal exercises. J Orthop Sports Phys Ther. 2010;40(5):265-76.

9. Imai A, Kaneoka K, Okubo Y et al. Trunk muscle activity during lumbar stabilization exercises on both a stable and unstable surface. J Orthop Sports Phys Ther. 2010;40(6):369-75.

10. Lehman GJ, Gilas D, Patel U. An unstable support surface does not increase scapulothoracic stabilizing muscle activity during push up and push up plus exercises. Man Ther. 2008;13(6):500-6.

11. Kong YS, Cho YH, ParkJW. Changes in the activities of the trunk muscles in different kinds of bridging exercises. J Phys Ther Sci. 2013;25(12): 1609-12.

12. Kang H, Jung J, Yu J. Comparison of trunk muscle activity during bridging exercises using a sling in patients with low back pain. J Sports Sci Med. 2012;11(3):510-5.

13. Garcia-Vaquero MP, Moreside JM, Brontons-Gil E et al. Trunk muscle activation during stabilization exercises with single and double leg support. J Electromyogr Kinesiol. 2012;22(3):398-406.
14. Stevens VK, Bouche KG, Mahieu NN et al. Trunk muscle activity in healthy subjects during bridging stabilization exercises. BMC Musculoskeletal Disorders. 2006;7:75.

15. Park SK, Kim JH. Effects of EMG-biofeedback training on total knee replacement patients' lower Extremity Muscle Activity and Balance. J Kor Phys Ther. 2013:25(2):81-7

16. Park HJ, Oh DW, Kim SY. Effects of integrating hip movements into bridge exercises on electromyographic activities of selected trunk muscles in healthy individuals. Man Ther. 2014;19(3):246-51.

17. Jang EM, Kim MH, Oh JS. Effects of a bridging exercise with hip adduction on the EMG activities of the abdominal and hip extensor muscles in females. J Phys Ther Sci 2013;25(9):1147-9.

18. Stevens VK, Coorevits PL, Bouche KG et al. The influence of specific training on trunk muscle recruitment patterns in healthy subjects during stabilization exercises. Man Ther. 2007;12(3):271-9.

19. Criswell, E. Cram's introduction to surface electromyography. Canada, Jones \& Bartlett Publishers, 2010:342-70.

20. Shumway-Cook A, Woollacott M. Normal postural control: Motor control translation research into clinical Practice. Lippincott Williams \& Wilkins. 2001:158-86.

21. Ryerson S, Levit K. Function movement reeducation: A contemporary model for stroke rehabilitation. Churchill Livingstone. 1997:1-14.

22. Bolgla LA, Uhl TL. Electromyographic analysis of hip rehabilitation exercises in a group of healthy subjects. J Orthop Sports Phys Ther. 2005; 35(8):487-94.

23. Haynes W. Core stability and the unstable platform device. J Bodyw Mov Ther. 2004;8(2):88-103. 